\title{
Validación de un cuestionario de clima organizacional para organizaciones de salud*
}

\section{Validation of a Questionnaire on Organizational Climate Intended to Health Organizations}

\section{Validação de um questionário de clima organizacional para organizações de saúde}

Fecha de recepción: 7 de marzo de 2018. Fecha de aprobación: 18deagostode 2018.

Fecha de publicación: 27 de mayo de 2019

DOI: https://doi.org/10.11144/Javeriana.rgps18-36.vcco

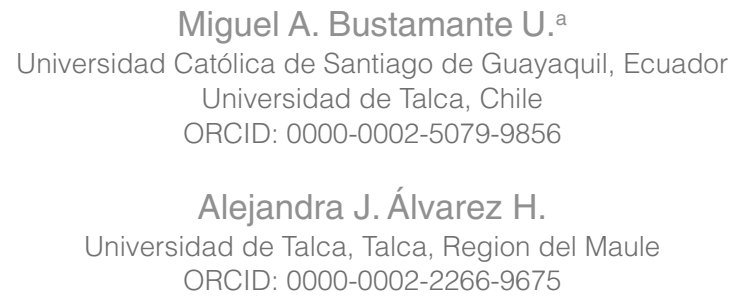

Como citar este artículo: Bustamante MA, Álvarez AJ. Validación de un cuestionario de clima organizacion-al para organizaciones de salud. Revista Gerencia y Políticas de Salud. 2019;18(36).

https://doi.org/10.11144/Javeriana.rgps18-36.vcco

Artículo de investigación

Autor de correspondencia. Correo electrónico: mabu@utalca.cl 


\section{Resumen}

El presente trabajo rediseña un cuestionario de clima organizacional para hospitales de alta complejidad validando sus dimensiones y reactivos en unidades de atención primaria de salud. Se determinaron dos grupos muestrales: en Chile 389 casos y en Ecuador 583. Se sensibilizaron los reactivos mediante análisis de cargas factoriales y comunalidad y se validaron con modelamiento por ecuaciones estructurales. Cuatro dimensiones sintetizan 27 reactivos validados. Las relaciones directas e inversas entre los factores concuerdan con lo planteado por la teoría, se confirma homocedasticidad de las matrices de varianza y covarianza y se obtienen índices de bondad de ajuste que confirman el modelo. Se confirma que la tensión interna y el desarrollo profesional influyen significativamente y de manera inversa sobre los factores gestión interna y organización y desempeño. Los ajustes realizados al modelo y los índices de bondad de ajuste confirman que el modelo se asemeja adecuadamente a la realidad.

Palabras clave: clima organizacional, validación multivariada, dimensiones del clima en APS, ecuaciones estructurales

\section{Abstract}

This work presents a redesign of the organizational climate questionnaire for high-complexity hospitals, by evaluating the hospital dimensions and reaction processes in the Primary Health Care (PHC) units. Two sample groups were used: 389 cases in Chile and 583 in Ecuador. The reaction processes were made sensitive according to an analysis of factor loads and commonalities and were then validated with a modelling based on structural equations. Four dimensions summarize the 27 reaction processes validated herein. Direct and inverse relations between the factors match the assumptions provided in the theory. The variance and covariance of the matrices confirmed that it is a homoscedastic model. The measures of goodness of fit validated the model. It is then confirmed that both the internal tension and the professional development influence significantly and inversely the internal management factors as well as the organization and performance. The adjustments done to the model and the goodness of fit measures confirmed that the model represents appropriately the reality.

Keywords: organizational climate, multivariate validation, climate dimensions in the PHC, structural equations

\section{Resumo}

O presente trabalho redesenha um questionário de clima organizacional para hospitais altamente complexos validando suas dimensões e reativos em unidades de atenção primária de saúde. Dois grupos de amostras foram determinados: no Chile 389 casos e no Equador 583. Sensibilizaram-se os reativos mediante análise de cargas fatoriais e comunalidade e validaram-se com modelagem de equações estruturais. Quatro dimensões sintetizam 27 reativos validados. As relações diretas e inversas entre os fatores concordam com o colocado pela teoria, confirma-se homocedasticidade das matrizes de variância e covariância e obtém-se índices de bondade de ajuste que confirmam o modelo. Confirma-se que a tensão interna e o desenvolvimento profissional influenciam significativamente e de maneira inversa sobre os fatores gestão interna e organização e desempenho. Os ajustes realizados no modelo e os índices de bondade de ajuste confirmam que o modelo semeia adequadamente à realidade.

Palavras chave: clima organizacional, validação multivariada, dimensões do clima em APS, equações estruturais 


\section{Introducción}

El clima organizacional es un constructo complejo de múltiples dimensiones (1) que tiene la capacidad de caracterizar una realidad organizacional (2) y definir escenarios (3) multidimensionales y complejos $(4,5)$. Como constructo complejo, se lo relaciona con la cultura organizacional, en cuanto hace referencia a un sistema de significados y a un patrón de creencias y expectativas compartidas por los miembros, las cuales norman y regulan las conductas individuales o colectivas en la organización (6).

Se lo define además como un conjunto de propiedades del ambiente de trabajo que directa o indirectamente inciden sobre las conductas $(7,8)$ y que pueden ser medidas por medio de percepciones (9). Estas percepciones cuantifican ítems y dimensiones del ambiente laboral (10), definen un patrón de interacciones de las personas en la organización (3) e identifican elementos intervinientes que contextualizan la realidad organizacional (11).

El clima organizacional sintetiza entonces las percepciones compartidas, autorreflexión y autoobservación de la experiencia organizacional $(12,13)$ y, aunque son inductores de conductas, no son necesariamente expresión directa de la realidad (10), sino tan solo caracterizaciones derivadas de percepciones que las personas asumen como una realidad. En esta misma línea, el diagnóstico del clima organizacional se constituye en una herramienta de primer orden en las instituciones de salud, especialmente en APS (2), puesto que permite medir además la satisfacción laboral (14).

En su carácter de herramienta para la gestión, se traduce en un cuestionario multifactorial que ha sido probado en diversas realidades $(12,13)$, por ejemplo, entre profesionales de enfermería (15), y ha confirmado su alta fiabilidad y consistencia interna a nivel de APS. Además, desde una perspectiva más bien de carácter prospectivo, es también un medio que estimula el desarrollo de acciones (16) orientadas hacia la misión y calidad en la gestión de las instituciones $(17,18)$.

Se afirma que puntuaciones altas de un cuestionario de clima predicen resultados positivos en unidades de atención primaria de salud (APS) (19) y que es vital propiciar la gestión eficaz de las unidades (20), por cuanto es preciso asegurar prestaciones de salud de calidad a los usuarios, lo que resulta vital para los sistemas sanitarios en el último decenio (21). Sin embargo, esto 
parece no ser suficiente puesto que, ante la existencia de déficit de personas en los servicios prestadores de salud (22), se precisa potenciar el sistema sanitario como un todo (23), y es aquí donde desempeña un rol importante el clima organizacional como constructo $(12,13)$.

A nivel teórico-práctico se han identificado aportaciones relevantes provenientes de los servicios de salud. Así, 83 investigaciones ratifican el enfoque multidimensional del clima organizacional (16). Adicionalmente, otros estudios han confirmado que las mediciones del clima son esenciales para el desarrollo de las instituciones de salud pública $(17,18)$.

Por otra parte, a nivel de la gestión interna de las organizaciones del sector salud, se ha llegado a identificar hasta 80 ítems influyentes en la conducta de un trabajador $(23,24)$. Es el caso de algunos estudios con directivos y funcionarios que ratifican el hecho de que, el saber y la experticia de los trabajadores, profesionales y jefaturas son factores clave para lograr el éxito de los individuos en términos personales y profesionales, así como para alcanzar las metas (25) y, en consecuencia, es a partir de estos logros que las organizaciones pueden generar bases sólidas para prestar servicios de salud de calidad a la comunidad (2).

Sobre la base de lo indicado, a nivel interno, el clima organizacional incide en la seguridad de los individuos (26) y se constituye en un factor clave en el sector prestador de servicios de salud $(11,27)$, por cuanto activa la motivación hospitalaria (28), potencia el desempeño de los empleados, así como eleva la satisfacción, compromiso y calidad de trabajo realizado $(4,5)$. Esto genera, por lo tanto, entornos laborales favorables $(29,30)$ que son percibidos por el estamento médico (31) y, dentro de las unidades $(32,33)$, por los estamentos de enfermería y de apoyo (34) y, por esta vía, se logra mejorar la calidad de atención y servicio entregado a los usuarios (35).

En síntesis, a efectos del presente trabajo, en Chile recientemente se presentó un instrumento que contiene 14 dimensiones y 71 reactivos en un cuestionario de clima organizacional (CCO) (13) que fue luego sensibilizado psicométricamente mediante análisis de factores exploratorio (AFE), simplificándolo a 10 dimensiones y 44 reactivos (CCOV) (12). El instrumento resultante de esta sensibilización permite medir de manera sistémica las percepciones del clima (18), así como derivar información clave para la 
toma de decisiones (17), por una parte, para verificar situaciones problema y, por otra, para descubrir posibilidades y recursos disponibles $(33,34) \mathrm{y}$, finalmente, determinar cursos de acción con los profesionales de la salud $(31,34)$, de manera que contribuyan efectivamente a mejorar el ambiente interno $(35,36)$ y el control de sus actividades $(37,38)$.

Sobre la base de los estudios mencionados, el presente trabajo asume el objetivo general de rediseñar el cuestionario de clima organizacional validado, $\operatorname{CCOV}(12)$, formulado originalmente para hospitales de alta complejidad, mediante su validación y modelamiento en unidades de APS.

\section{Material y métodos}

Se tomó como referencia el Cuestionario de Clima Organizacional Validado $\operatorname{CCOV}(12)$, que mide 10 dimensiones e integra 44 reactivos medidos mediante escala Likert de cinco puntos, donde el valor 1 indica estar "totalmente en desacuerdo" y el valor 5 señala estar "totalmente de acuerdo". Este tipo de escala, denominada policórica, permite asumir que es posible superar la eventual no normalidad de los datos (39).

\section{Muestras y validaciones}

Para evaluar la consistencia interna de las escalas, se tuvieron en consideración algunos criterios como número de ítems por factor y comunalidades de dichos ítems. Se consideró que la estabilidad de los resultados se reduce con muestras menores (39) y que muestras superiores son más seguras y modelables porque soportan un mayor número de ítems por factor (40).

Se optó por sensibilizar la muestra mediante criterios que sugieren un tamaño de muestra mínimo (n), analizando los criterios de la ratio personas/ ítems (n/p), denominada regla 10:1 y regla 5:1, que exigen entre diez y cinco sujetos por variable, y un tamaño no menor de cien casos, para determinar una muestra aceptable (41). Además, considerando que el tamaño de la muestra interactúa con la naturaleza de los datos, la matriz input del análisis factorial exploratorio (AFE) y la homogeneidad de la muestra, se acogieron algunas sugerencias analíticas de saturaciones y comunalidades altas superiores al mínimo $(0,30)$ y se optó por alcanzar una muestra por encima de los quinientos casos para obtener estimaciones más precisas (42). 
La muestra se calculó a priori mediante el programa Gpower 3.1, sobre la base de los parámetros de poder y tamaño de efecto para dos grupos con diferentes inclinaciones (41). Para ello, se consideró una inclinación de 0,015 , un error probabilístico de 0,05 y una confiabilidad o impulso probabilístico de 0,95 , un ratio de muestras igual a 2 , una estándar residual de 0,5 y una desviación estándar de la muestra de 7 para la muestra 1 y de 20 para la muestra 2.

A partir de lo señalado, se determinó una muestra estimada total de 942 casos, 314 para la muestra en Chile y de 628 para la muestra en Ecuador, todo ello considerando una confiabilidad de 0,9503815 , un parámetro de centralidad de 3,1622289 y un valor crítico de 1,9624963.

Posteriormente, se procedió a verificar la muestra post hoc revisando datos perdidos y valores atípicos, hasta llegar a una muestra corregida y depurada $(41,42)$. En consecuencia, se definieron 389 casos para la muestra chilena y 583 casos para la ecuatoriana, que fueron ajustadas a los parámetros de centralidad y arrojaron los valores de 3,9822939, valor crítico T de 1,9624177, valor Df 968 y un error probabilístico menor al estimado para la muestra a priori $(0,05)$, la cual resultó ser post hoc de 0,022 (1- 0,9782239).

En síntesis, la muestra chilena es superior a la estimada a priori (314) y la muestra ecuatoriana es levemente inferior a ella (628); sin embargo, el error muestra total post hoc es de tan solo $2,2 \%$.

\section{Validación del instrumento}

El instrumento CCOV (12) fue debidamente piloteado en Ecuador y en Chile, frente a quince trabajadores-funcionarios de cada realidad y no pertenecientes a la muestra final del estudio (42). Los sujetos de la muestra fueron seleccionados como respondientes normales del contexto APS objeto de la investigación (43).

Se verificó comprensión, lenguaje, formato, instrucciones y escalas utilizadas y, considerando los giros idiomáticos propios de cada país, se corrigieron algunos aspectos lingüísticos del cuestionario. Además, el instrumento fue revisado por un grupo de expertos conformado por ocho directores de APS tanto en Chile como en Ecuador, quienes no hicieron alcances mayores a esta nueva versión del instrumento, confirmando la unidireccionalidad de 
las proposiciones y la validación del lenguaje utilizado en las afirmaciones. Además, el equipo de investigación validó la consistencia del constructo en relación con los modelos teóricos consultados, verificando redacción y estructura semántica de cada reactivo $(31,44)$.

Finalmente, por el número de casos recogidos, 389 en Chile y 583 en Ecuador, se estima que es factible determinar un modelo correctamente especificado que resulte consistente, por una parte, utilizando variables categóricas Likert de cinco puntos para ser analizadas como variables discretas y, por otra, porque una escala policórica de cinco puntos permite asumir que es factible superar la trampa de la multicolinealidad perfecta de las variables dicotómicas (44) y contribuye a superar eventuales riesgos de no normalidad de los datos $(45,46)$.

\section{Procedimientos}

El CCOV (12) modificado se aplicó secuencialmente en ambos países y, en atención a que las unidades de atención de salud de APS son naturalmente pequeñas y concentran un número bajo de trabajadores, se optó por el método de censo.

En Chile el trabajo de campo se realizó durante una semana del mes de octubre de 2016 y se accedió a los funcionarios de APS de cuatro comunas de la séptima región del Maule, alcanzando una tasa de respuesta de $92 \%$.

En Ecuador se aplicó en octubre de 2017 en los centros prestadores de salud primaria de nueve localidades, denominadas parroquias, de la ciudad de Guayaquil, y alcanzó una tasa de respuesta de 90\% (43).

Ambas aplicaciones se realizaron en las propias oficinas de los sujetos entrevistados y por entrevistadores debidamente entrenados por los propios investigadores (42) quienes, con el objetivo de asegurar la confidencialidad de la información, optaron por un cuestionario anónimo bajo el principio de consentimiento informado. La duración promedio de respuesta fue de aproximadamente 20 a 30 minutos.

\section{Procesamiento de datos}

Se realizaron algunos pasos previos al procesamiento de datos. Se verificó la calidad de ambas muestras mediante el análisis de caja de Statistical 
Package for the Social Sciences (SPSS), para determinar casos perdidos o atípicos y asegurar la calidad de los datos recogidos.

En una primera fase, se aplicó a los datos chilenos el análisis de componentes principales (ACP) para la generación de los primeros constructos de fácil interpretación con ítems, dimensiones y cargas factoriales susceptibles de ser modelados (40). Seguidamente, se exploró y determinó la estructura factorial subyacente a los ítems mediante análisis factorial exploratorio (AFE), asumiendo que las medidas de los reactivos (ítems) son reflectivas o manifestaciones de los constructos subyacentes o latentes que se busca medir (47). Así mismo, considerando que AFE amplía las opciones de análisis de variables relacionadas que pudiesen pasar inadvertidas cuando se aplica rotación ortogonal, se empleó la metodología propuesta por Baño (48), asumiendo interdependencia de factores y rotación oblicua (direct oblimin) $(40,49)$.

En una segunda fase, se procedió a confirmar la estructura factorial determinada en la muestra ecuatoriana, esta vez mediante análisis de factores confirmatorio (AFC), lo que se realizó utilizando métodos inferenciales progresivamente más exigentes $(45,46)$. Consecuente con lo señalado, dado que el análisis AFC implica mayores restricciones, se puso a prueba y se confirmó la estructura del instrumento CCOV (12), validándolo en la muestra APS mediante los índices de ajuste comparativo (comparative fit index) CFI, índice de bondad de ajuste (goodness of fit index) GFI e índice ajustado de bondad de ajuste (adjusted goodness of fit index) AGFI (49), todos los cuales se ubican entre 0 y 1 , donde 1 indica un ajuste perfecto.

\section{Extracción de factores}

La extracción de factores se realizó aplicando la matriz de correlación Pearson, métodos de análisis paralelo (PA) y se ejecutó análisis factorial robusto (robust factor analysis) mediante el método de mínimos cuadrados no ponderados (ULS), ya que este método permite asumir y superar adecuadamente la eventual ocurrencia de no normalidad de los datos. Por otro lado, siguiendo las especificaciones de Pérez y Medrano (50) en la selección de reactivos, se consideraron índices mínimos de comunalidad $(\geq 0,3)$ y de cargas factoriales $(\geq 0,5)$, con el fin de descartar reactivos que posean baja correlación (51). 
Se verificó la confiabilidad del instrumento mediante alfa de Cronbach ( $\alpha$ $\geq 0,8)(52)$, para asegurar consistencia, estabilidad y precisión de los constructos del modelo en estudio. Además, se determinó el ídice Omega $(' \Omega)$ de McDonald's que determina la confiabilidad, previa medición de la fuerza de asociación entre los ítems. También se analizó la medida de adecuación KMO $(\geq 0,8)(53)$ y la prueba de esfericidad de Barlett (48), además de las gráficas de sedimentación (40) y el porcentaje de varianza explicada (54).

\section{Análisis factorial confirmatorio}

Ejecutados los pasos previos al modelamiento de ecuaciones estructurales SEM sugeridos por Kaplan (51), se aplicó análisis factorial confirmatorio (AFC) para ratificar las variables latentes del modelo (55).

Se optó por sensibilizar la reducción de dimensiones mediante análisis de comunalidad y cargas factoriales y se buscó retener los factores con mayor varianza explicada significativa (54) y evitar la aparición de saturaciones mayores que la unidad y varianzas de error negativas.

Los análisis de bondad de ajuste de esta fase del análisis se realizaron considerando el índice absoluto estadístico-radio de verosimilitud de chi-cuadrado (CMIN/DF $\leq 3)$, (56) el índice de bondad de ajuste GFI $(\geq 0,9)(57)$, el índice de error de aproximación cuadrático medio RMSEA $(\leq 0,05)(58)$ y el índice de error cuadrático medio RMR $(\approx 0),(59)$, además de las medidas de ajuste incremental índice ajustado de bondad AGFI $(\leq 0,8)(57)$ el índice no normado de ajuste o índice de Tucker Lewis TLI $(\leq 0,9)(60)$ y el índice normado de ajuste NFI $(\leq 0,9)(59,61)$.

En la aplicación del método de ecuaciones estructurales (exploratory structural equation modeling) ESEM, como alternativa semiconfirmatoria, $(62,63)$ se consideraron no menos de tres marcadores (ítems) por factor si los factores no están correlacionados, y como mínimo dos marcadores por factor si los factores están correlacionados. Además, se utilizaron los métodos de procesamiento (50) matriz de correlaciones producto-momento de Pearson y matriz de varianza-covarianza para prever y superar eventuales efectos de asimetría de las distribuciones de los ítems (40). 
Finalmente, se asume que opciones de respuesta categóricas en escala Likert de cinco puntos o más, se comportan de manera aproximadamente normal (39) y, por lo tanto, no derivan en relaciones no lineales. Se utilizó además el método de rotación Direct Oblimin para variables policóricas y se acogieron las recomendaciones de asimetría y curtosis para evitar el impacto negativo de la asimetría que interactúa con el tamaño de la muestra y el número de ítems que definen cada factor (64).

Por otra parte, se dieron por superados los riesgos de error de la muestra y error por aproximación (40), dado que la muestra total depurada para el estudio alcanza los 972 casos (389 en Chile y 583 en Ecuador) y los ítems se miden mediante escalas policóricas, lo que permite asumir que se supera razonablemente el supuesto de normalidad de los ítems (39) para alcanzar resultados satisfactorios (64-66).

\section{Uso de software}

El análisis y procesamiento de los datos se realizó mediante los software SPSS V20, para los análisis descriptivos de la muestra (43, y Factor.10.4.win64, (67-69) para los análisis psicométricos de confiabilidad de reactivos del instrumento.

Para el modelamiento mediante ecuaciones estructurales se utilizó AMOS de SPSS (70), aplicando los métodos de máxima verosimilitud (ML), mínimos cuadrados no ponderados (ULS) y mínimos cuadrados generalizados (GLS). Además, a fin de reducir los efectos de la varianza (42), se estandarizaron los datos (43) para realizar comparaciones relevantes.

\section{Resultados}

A continuación, se presentan los resultados de la investigación, comenzando con el análisis descriptivo de la muestra y el análisis de las dimensiones del clima organizacional que se ratifican en términos de los ítems que los conforman. Seguidamente, en la fase inferencial del análisis se detalla la validez convergente confirmada por el análisis de factores exploratorio (AFE), 
lo que da pie al modelamiento con ecuaciones estructurales respectivo. Se finaliza el estudio con el modelo de medida y estructural, el cual es confirmado mediante los índices de bondad de ajuste del modelo determinado.

\section{Análisis descriptivo}

Del total de funcionarios encuestados de la muestra chilena (389), el $71 \%$ corresponde a mujeres, el $57,6 \%$ es soltero, el $35 \%$ es casado. En términos de las edades, la mayor proporción, que alcanza a 45,8\%, tiene entre 26 y 35 años. En educación, el 47,6\% tiene educación superior completa y el $31,9 \%$ tiene educación técnica completa. Respecto de sus empleos, un 32,6\% pertenece a la categoría de técnicos de nivel superior, el $32,1 \%$ pertenece a la categoría de servicios profesionales. Además, el 49,1\% tiene entre uno a cinco años de servicio, el 55,5\% tiene contrato indefinido y el 33,2\% tiene contrato a plazo fijo.

La muestra ecuatoriana (583) evidencia que la mayoría es de sexo femenino $(70 \%)$, casi la totalidad $(98,1 \%)$ pertenece a las unidades de atención ambulatoria. En términos de empleo, se identificó con contrato definitivo el 58,7\%. Respecto de las edades de la muestra, las mayores frecuencias se registran a partir de los 26 años. Entre 26 y 34 años se observa un 29,4\% y entre 35 y 43 años un 23,1\%; finalmente, con más de 66 años se registra un $3 \%$.

\section{Análisis de dimensiones del clima organizacional}

Con el propósito de comparar de manera pertinente los cuestionarios en análisis (CCO y CCOV), se estandarizaron los resultados promedio de las dimensiones. Se observa (tabla 1) que el cuestionario de clima organizacional original (CCO) (13) cuenta con catorce dimensiones. Cinco se ubican por debajo del promedio estándar (recompensa, riesgo, administración del conflicto, comunicación, equipos y distribución de personas y material) y nueve por encima del cero estandarizado (estructura, responsabilidad, calidez, apoyo, identidad, estilo de supervisión, motivación laboral, estabilidad laboral, oportunidad de desarrollo). 
Tabla 1. Comparación de dimensiones de los cuestionarios CCO y CCOV

\begin{tabular}{|c|c|c|c|c|c|c|c|}
\hline \multirow{8}{*}{ 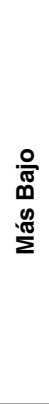 } & \multirow{2}{*}{$\begin{array}{l}\text { Dimensiones CCO } \\
\text { Hospitales } \\
\text { Recompensa }\end{array}$} & \multirow{2}{*}{$\begin{array}{l}\text { Medias } \\
3,00\end{array}$} & \multirow{2}{*}{$\begin{array}{l}\mathbf{Z} \\
-0,19\end{array}$} & \multicolumn{3}{|c|}{ Dimensiones CCOV } & Medias \\
\hline & & & & \multicolumn{2}{|c|}{ Iniciativa participativa } & 3,26 & $-0,02$ \\
\hline & Riesgo & 3,00 & $-0,18$ & \multicolumn{2}{|c|}{ Apoyo al emprendimiento } & 3,21 & $-0,06$ \\
\hline & Administración del conflicto & 2,57 & $-1,30$ & \multicolumn{2}{|c|}{$\begin{array}{l}\text { Equipos y distribución de } \\
\text { personas y material }\end{array}$} & 2,97 & $-0,26$ \\
\hline & Comunicación & 2,94 & $-0,33$ & \multicolumn{2}{|c|}{ Organización y desempeño } & 3,06 & $-0,19$ \\
\hline & \multirow{3}{*}{$\begin{array}{l}\text { Equipos y distribución de } \\
\text { personas y material }\end{array}$} & \multirow{3}{*}{2,01} & $-2,74$ & \multicolumn{2}{|c|}{ Estabilidad laboral } & 3,12 & $-0,14$ \\
\hline & & & & \multicolumn{2}{|c|}{ Comunicación y desarrollo } & 3,26 & $-0,02$ \\
\hline & & & & \multicolumn{2}{|c|}{ Colaboración y buen trato } & 3,54 & 0,20 \\
\hline \multirow{9}{*}{$\begin{array}{l}\frac{0}{4} \\
\frac{0}{4} \\
\frac{90}{\Sigma} \\
\Sigma\end{array}$} & Estructura & 3,27 & 0,51 & & & & \\
\hline & Responsabilidad & 3,32 & 0,66 & & & & \\
\hline & Calidez & 3,19 & 0,31 & & & & \\
\hline & Apoyo & 3,17 & 0,25 & & & & \\
\hline & Identidad & 3,56 & 1,27 & \multicolumn{2}{|c|}{ Desarrollo profesional } & 3,30 & 0,01 \\
\hline & Estilo de supervisión & 3,19 & 0,31 & & & & \\
\hline & Motivación laboral & 3,44 & 0,96 & \multicolumn{2}{|c|}{ Motivación laboral } & 3,57 & 0,23 \\
\hline & Estabilidad laboral & 3,22 & 0,37 & & & & \\
\hline & Oportunidad de desarrollo & 3,11 & 0,09 & \multicolumn{2}{|c|}{ Autonomía y profesionalismo } & 3,73 & 0,36 \\
\hline \multicolumn{8}{|c|}{ Confiabilidad de las dimensiones CCOV } \\
\hline N. ${ }^{\circ}$ & \multicolumn{3}{|l|}{ Dimensiones } & $\alpha$ & Media & $\mathrm{Z}$ & \\
\hline 1 & \multicolumn{3}{|l|}{ Colaboración y buen trato } & 0,920 & 3,54 & 0,20 & \\
\hline 2 & \multicolumn{3}{|l|}{ Motivación laboral } & 0,920 & 3,57 & 0,23 & \\
\hline 3 & \multicolumn{3}{|l|}{ Iniciativa participativa } & 0,890 & 3,26 & $-0,02$ & \\
\hline 4 & \multicolumn{3}{|l|}{ Desarrollo profesional } & 0,499 & 3,30 & 0,01 & \\
\hline 5 & \multicolumn{3}{|l|}{ Apoyo al emprendimiento } & 0,583 & 3,21 & $-0,06$ & \\
\hline 6 & Equipos y distribución de per & has y mater & & 0,822 & 2,97 & $-0,26$ & \\
\hline 7 & Organización y desempeño & & & 0,842 & 3,06 & $-0,19$ & \\
\hline 8 & Estabilidad laboral & & & 0,826 & 3,12 & $-0,14$ & \\
\hline 9 & Comunicación y desarrollo & & & 0,892 & 3,26 & $-0,02$ & \\
\hline 10 & Autonomía y profesionalismo & & & 0,657 & 3,73 & 0,36 & \\
\hline Con & fiabilidad (44 elementos) & & 0,950 & & & & \\
\hline Mec & ia general (44 elementos) & & & & 3,29 & & \\
\hline Vari & anza general & & & & 1,49 & & \\
\hline Des & viación estándar general & & & & 1,22 & & \\
\hline $\mathrm{KMC}$ & y prueba de Bartlett & & & 0,92468 & & & \\
\hline Mec & ida de adecuación de la muest & de Kaiser-1 & Meyer-C & kin & & & 0,955 \\
\hline & Chi-cuadrado a & oximado & & & & & 10828,089 \\
\hline & & Prueba de & esferic & dad de B & tlett & $\mathrm{gl}$ & 946 \\
\hline & & & & & & Sig. & 0,000 \\
\hline
\end{tabular}

$\mathbf{Z}=$ Valor estandarizado

$\boldsymbol{\alpha}=$ Alfa de Cronbach

Fuente: elaboración propia 
El cuestionario de clima organizacional validado (CCOV) (12), que registra diez dimensiones, presenta siete de ellas ubicadas por debajo del cero estándar (iniciativa participativa, apoyo al emprendimiento, equipos y distribución de personas y material, organización y desempeño, estabilidad laboral, comunicación y desarrollo, y colaboración y buen trato). Las tres dimensiones restantes alcanzan valores positivos a la derecha de la curva normal (desarrollo profesional, motivación laboral y autonomía y profesionalismo). En consecuencia, existen dos coincidencias. Por debajo del promedio estándar se ubica la dimensión equipos y distribución de personas y material y por encima se encuentra la dimensión motivación laboral.

\section{Confiabilidad del instrumento}

Respecto de la confiabilidad, se observa que a nivel general el coeficiente alfa de Cronbach de CCOV alcanza un índice 0,955 que ratifica la consistencia integral del instrumento (40).

Sin embargo, siete dimensiones muestran un alfa $\geq 0,8$ (colaboración y buen trato, motivación laboral, iniciativa participativa, equipos y distribución de personas y material, organización y desempeño, estabilidad laboral y comunicación y desarrollo), lo que ratifica una buena fiabilidad. Dos dimensiones alcanzan un alfa moderado de entre 0,5 y 0,8 (desarrollo profesional y apoyo al emprendimiento), y una dimensión presenta un alfa $\leq 0,5$, calificado de bajo (autonomía y profesionalismo) $(50,51)$.

\section{Validez convergente mediante análisis de factores exploratorio}

La validez convergente (tabla 2) se confirma mediante el agrupamiento coherente de ítems en dimensiones representativas, en tanto que la persistencia de los reactivos se verifica mediante el análisis de las comunalidades.

Los 29 ítems efectivamente confirmados (tabla 2) fueron ratificados mediante análisis para variables policóricas, análisis paralelo, robusto y con rotación oblicua (direct oblimin), realizado mediante el programa FACTOR, y se obtuvieron los índices de Kurtosis de 1123,817 con un valor P ,0000** significativo al $5 \%$.

La verificación de pertinencia y medida de adecuación de la muestra de Kaiser-Meyer-Olkin (KMO) es de 0,92056 (0,921 - 0,925) y la prueba de esfericidad de Bartlett alcanza a 10901,7 ( $\mathrm{df}=406 ; \mathrm{P}=, 000010)$, que resultó significativa al 0,000 (1\%). 
Los índices que determinan la varianza explicada que alcanza cada uno de los factores determinados mediante AFE (real-data \% of variance, significativos, p: ,000), se expresa porcentualmente, en forma decreciente e indicando su respectiva significación $(* *)$.

El factor 1 alcanza un índice de 35,7\%** de varianza explicada significativa al $5 \%$, el factor 2 presenta un $18,9 \% * *$ de varianza explicada y significativa también al 5\%, el factor 3 entrega como resultados un 6,6\%* de varianza explicada y significativa al $10 \%$ y el factor 4 obtiene un 5,4\% de varianza explica aunque resulta no significativa.

En consecuencia, totalizando la varianza de los cuatro factores determinados se llega a un 66,6\% de varianza explicada con una $(\alpha)$ de Cronbach de 0,902126 , lo que confirma la confiabilidad y consistencia general del instrumento utilizado en el análisis.

Así mismo, para ratificar la estructura general del instrumento, se calculó el índice' $\Omega$ de McDonald's, y se obtuvo un valor de $0,820738 \geq 0,80$, resultado que se compara favorablemente con el $\alpha$ de Cronbach y confirma los resultados.

Seguidamente, se midió el error de aproximación cuadrático medio (root mean square error of approximation) RMSEA, que alcanza un resultado de 0,084 al $95 \%$, con un nivel de confianza en el rango de intervalo determinado (0,0096 - 0,1086). Adicionalmente, se midió el índice raíz cuadrada del valor medio de los cuadrados de los residuos (root mean square of residuals) RMSR, que alcanzó un valor de $0,0416 \leq 0,05$, con un $95 \%$ de nivel de confianza y ubicado en el intervalo resultante $(0,011-0,050)$.

En síntesis, los indicadores de confiabilidad $\alpha$ de Cronbach y' $\Omega$ de McDonald, así como los índices de bondad de ajuste, error de aproximación (RMSEA) y raíz del cuadro de los residuos (RMSR) ratifican los factores del constructo obtenidos mediante AFE.

Sin embargo, del análisis AFE se observa la eliminación de los reactivos V22 y V23 que, aun cuando poseían valores de comunalidad adecuados $(0,711065$ y 0,76842 , respectivamente), no mostraron cargas factoriales al nivel exigido que fue de al menos 0,5 puntos; en consecuencia, los reactivos sensibilizados y persistentes de clima organizacional confirmados resultaron ser finalmente 27 ítems (tabla 2). 
Tabla 2. Matriz de componentes rotados del análisis de factores AFE

\begin{tabular}{|c|c|c|c|c|c|}
\hline 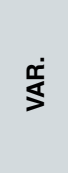 & 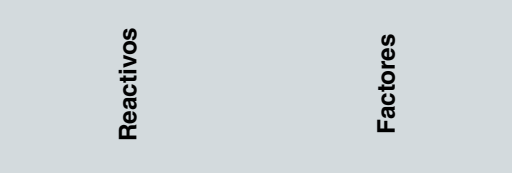 & $\overline{4}$ & $\stackrel{N}{u}$ & $\stackrel{m}{\Perp}$ & 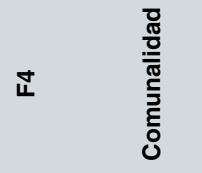 \\
\hline V 11 & $\begin{array}{l}\text { Cuando tengo que hacer un trabajo } \\
\text { difícil puedo contar con la ayuda de mis } \\
\text { compañeros o colegas }\end{array}$ & 0,741 & & & 0,642309 \\
\hline V 12 & $\begin{array}{l}\text { Los jefes entregan información a su personal } \\
\text { respecto de su desempeño laboral }\end{array}$ & 0,704 & & & 0,763144 \\
\hline$\vee 13$ & $\begin{array}{l}\text { Los trabajadores de la salud saben lo que } \\
\text { sus jefes esperan de ellos }\end{array}$ & 0,771 & & & 0,797135 \\
\hline V 14 & $\begin{array}{l}\text { Los jefes promueven las buenas relaciones } \\
\text { humanas entre las personas de la institución }\end{array}$ & 0,742 & & & 0,820957 \\
\hline V 15 & $\begin{array}{l}\text { Los jefes de esta institución tratan con } \\
\text { respeto a sus colaboradores }\end{array}$ & 0,721 & & & 0,936705 \\
\hline V 16 & $\begin{array}{l}\text { Cuando tengo que hacer un trabajo difícil } \\
\text { puedo contar con la ayuda de mi(s) jefe(s) }\end{array}$ & 0,709 & & & 0,801238 \\
\hline V 18 & $\begin{array}{l}\text { En esta institución los colaboradores están } \\
\text { comprometidos con su trabajo }\end{array}$ & 0,751 & & & 0,852860 \\
\hline V 19 & $\begin{array}{l}\text { Las personas de esta institución se } \\
\text { esfuerzan por desarrollar eficientemente su } \\
\text { labor }\end{array}$ & 0,780 & & & 0,923338 \\
\hline V 20 & $\begin{array}{l}\text { Las personas en esta institución muestran } \\
\text { interés por el trabajo que realizan }\end{array}$ & 0,787 & & & 0,838461 \\
\hline V 21 & $\begin{array}{l}\text { Aquí se da facilidad para que cualquier } \\
\text { colaborador pueda presentar una idea nueva }\end{array}$ & 0,521 & & & 0,756130 \\
\hline V 22 & $\begin{array}{l}\text { Los jefes son comprensivos cuando uno } \\
\text { comete un error }\end{array}$ & & & & 0,711065 \\
\hline V 23 & $\begin{array}{l}\text { Uno progresa en esta institución si tiene } \\
\text { iniciativa }\end{array}$ & & & & 0,707734 \\
\hline V 45 & $\begin{array}{l}\text { Me preocupa que por una reorganización en } \\
\text { la institución se vea afectada la estabilidad } \\
\text { de mi trabajo }\end{array}$ & & 0,854 & & 0,892209 \\
\hline V 46 & $\begin{array}{l}\text { Me preocupan los efectos que puedan } \\
\text { tener sobre la estabilidad de mi empleo } \\
\text { los cambios de método de trabajo o la } \\
\text { automatización en esta institución }\end{array}$ & & 0,898 & & 0,799973 \\
\hline V 47 & $\begin{array}{l}\text { Creo que en cualquier momento puedo } \\
\text { perder el trabajo en esta institución }\end{array}$ & & 0,696 & & 0,751698 \\
\hline V 28 & $\begin{array}{l}\text { En esta institución no se dan las } \\
\text { oportunidades para desarrollar las destrezas } \\
\text { y habilidades personales }\end{array}$ & & & 0,626 & 0,720799 \\
\hline V 29 & $\begin{array}{l}\text { Los trabajadores de esta institución creen } \\
\text { más en el rumor que surge sobre un cierto } \\
\text { hecho que en la información oficial }\end{array}$ & & & 0,633 & 0,620652 \\
\hline
\end{tabular}




\begin{tabular}{|c|c|c|c|c|c|c|}
\hline 宅 & 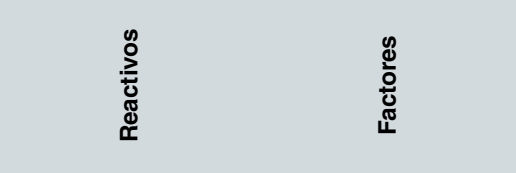 & $\bar{\leftarrow}$ & $\underset{\sim}{\sim}$ & $\stackrel{m}{\Perp}$ & 芒 & 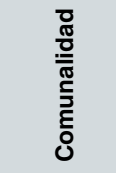 \\
\hline V 30 & $\begin{array}{l}\text { Esta institución se caracteriza por un } \\
\text { ambiente de trabajo tenso }\end{array}$ & & & 0,723 & & 0,824419 \\
\hline V 31 & $\begin{array}{l}\text { En esta institución se nos mantiene } \\
\text { desinformados sobre materias que } \\
\text { deberíamos saber }\end{array}$ & & & 0,688 & & 0,689268 \\
\hline V 32 & $\begin{array}{l}\text { El ambiente en esta institución no es propicio } \\
\text { para desarrollar nuevas ideas }\end{array}$ & & & 0,690 & & 0,749314 \\
\hline V 33 & $\begin{array}{l}\text { Las nuevas ideas que aporta el personal no } \\
\text { son bien acogidas por los jefes }\end{array}$ & & & 0,757 & & 0,800806 \\
\hline V 34 & $\begin{array}{l}\text { En esta institución existen demasiadas } \\
\text { críticas }\end{array}$ & & & 0,684 & & 0,702535 \\
\hline V 37 & $\begin{array}{l}\text { A veces trabajamos en forma desorganizada } \\
\text { y sin planificación }\end{array}$ & & & 0,670 & & 0,603609 \\
\hline$\vee 25$ & $\begin{array}{l}\text { La dirección de esta institución se preocupa } \\
\text { por las personas, de cómo se sienten y de } \\
\text { sus problemas }\end{array}$ & & & & 0,598 & 0,706842 \\
\hline V 41 & $\begin{array}{l}\text { En esta institución los trabajos están bien } \\
\text { asignados y organizados }\end{array}$ & & & & 0,618 & 0,893804 \\
\hline V 42 & $\begin{array}{l}\text { La capacidad es el criterio básico para } \\
\text { asignar tareas en esta institución }\end{array}$ & & & & 0,510 & 0,619879 \\
\hline V 48 & $\begin{array}{l}\text { Existe una buena comunicación entre la } \\
\text { dirección y los trabajadores }\end{array}$ & & & & 0,847 & 0,775112 \\
\hline V 49 & $\begin{array}{l}\text { Todo lo que hay que hacer está claro, } \\
\text { porque se nos explica bien y oportunamente }\end{array}$ & & & & 0,752 & 0,806839 \\
\hline V 51 & $\begin{array}{l}\text { Existe una buena comunicación entre las } \\
\text { diferentes unidades y servicios que trabajan } \\
\text { generalmente juntos }\end{array}$ & & & & 0,702 & 0,704542 \\
\hline \multicolumn{2}{|c|}{$\begin{array}{l}\text { Porcentaje de varianza explicada por } \\
\text { los valores propios }\end{array}$} & 0,32991 & 0,17474 & 0,06251 & 0,05234 & \\
\hline \multicolumn{2}{|c|}{ Acumulado } & 0,32991 & 0,50465 & 0,56722 & 0,61956 & \\
\hline \multicolumn{2}{|c|}{ Porcentaje de varianza real } & $35,7^{\star \star}$ & $18,9^{\star *}$ & $6,6^{*}$ & 5,4 & \\
\hline \multicolumn{2}{|c|}{ Acumulado } & 35,1 & 52,8 & 59,2 & 66,6 & \\
\hline \multicolumn{2}{|c|}{ Alfa $(\alpha)$ de Cronbach } & & & & 0,902126 & \\
\hline
\end{tabular}

$\left({ }^{\star *}\right)=$ Significación al $5 \%$

$\left(^{\star}\right)=$ Significación al 10\%

Fuente: elaboración propia

\section{Modelamiento con ecuaciones estructurales}

Los factores resultantes del análisis AFE, gestión interna (FC1), estabilidad laboral (FC2), tensión interna y desarrollo profesional (FC3) y organización y desempeño (FC4) ponen en evidencia el rol de todas estas dimensiones 
dentro del clima organizacional $(1,2,31)$ y fueron sometidos al análisis de ecuaciones estructurales (ESEM) utilizando los métodos ML, ULS y GLS (40).

Como primer paso, se realizó el análisis de covarianza, equivalente a efectuar correlaciones, y se determinó que existen relaciones entre los cuatro constructos. Se observa que gestión interna (FC1) se relaciona de manera negativa o inversa $(-0,01)$ con tensión interna y desarrollo profesional (FC3) y que esta última se relaciona también de manera inversa o negativa $(-0,01)$ con organización y desempeño (FC4). Las demás relaciones muestran signo positivo directamente proporcional entre los factores del clima.

Como segundo paso, se realizó el análisis de varianza (figura 1) que especifica las relaciones de causalidad para determinar si el factor organización y desempeño (FC4) se ve influido por los otros tres factores que componen el modelo de clima organizacional. Se definió como variable exógena independiente el constructo gestión interna (FC1), variable endógena dependiente la dimensión organización y desempeño (FC4) y las dimensiones mediadoras estabilidad laboral (FC2) y tensión interna y desarrollo profesional (FC3).

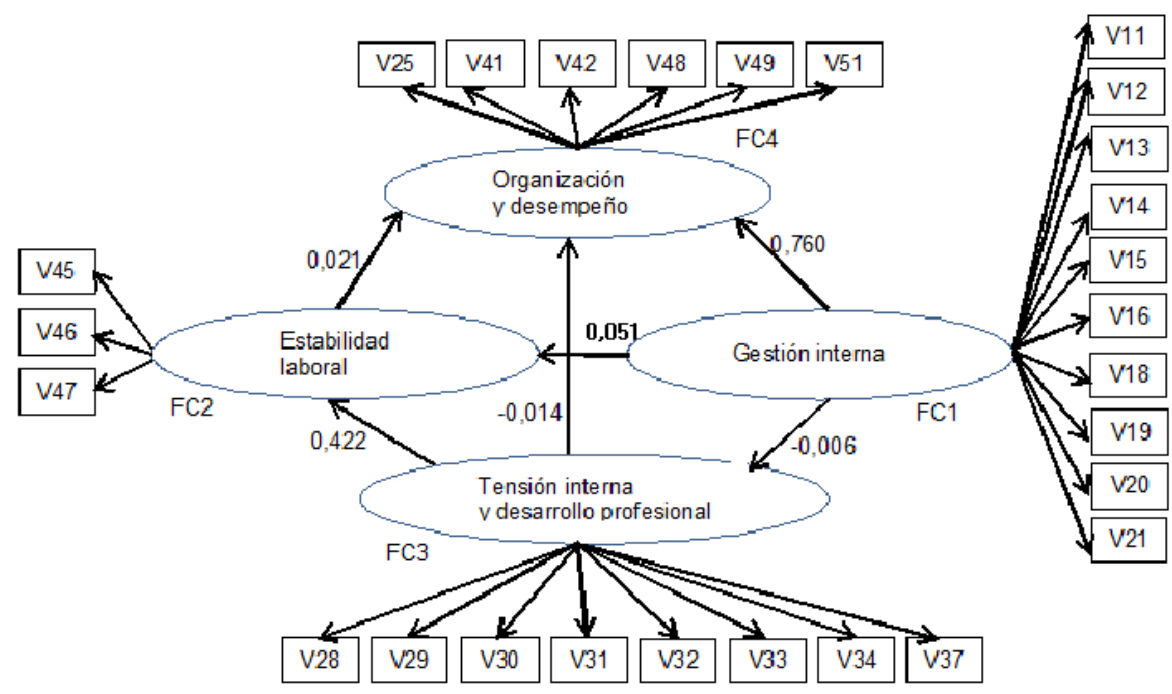

Figura 1. Modelamiento estructural estandarizado, relaciones de varianzas por el método ML Fuente: elaboración propia 
Se observa en la figura 1 que el constructo FC1, gestión interna, impacta en forma directa y en una mayor proporción al factor FC4, organización y desempeño $(\mathrm{FC} 4 \leftarrow \mathrm{FC} 1=0,76)$, de manera que variaciones en la gestión interna, alteran progresivamente y en forma positiva la organización y el desempeño.

Además, al igual que en el modelo de covarianzas, las relaciones de FC1 gestión interna, con FC3 tensión interna y desarrollo profesional, se vinculan mediante un índice negativo $(\mathrm{FC} 3 \leftarrow \mathrm{FC} 1=-0,006)$, estableciendo una relación inversa entre estas dimensiones de clima organizacional.

Finalmente, FC3 tensión interna y desarrollo profesional se relaciona con FC4 organización y desempeño, también mediante un índice negativo o inverso (FC4 $\leftarrow$ FC3 $=-0,014$ ), lo cual evidencia que un incremento en tensión interna y desarrollo profesional impacta negativamente sobre organización y desempeño.

La tabla 3 muestra los parámetros estimados del modelo de factores de clima organizacional calculados mediante los métodos máxima verosimilitud (ML), mínimos cuadrados no ponderados (ULS) y mínimos cuadrados generalizados (GLS) (40).

Adicionalmente, es preciso destacar que los coeficientes $\beta_{1}$ y $\beta_{2}$ para cada una de las variables que conforman los cuatro factores de clima organizacional alcanzan índices normalizados en rangos pertinentes que van entre 0 y 1 . 
Tabla 3. Parámetros estimados del modelo de factores de clima organizacional

\begin{tabular}{lllllll}
\hline \multirow{2}{*}{ Modelo de varianzas } & ML & \multicolumn{3}{c}{ ULS } & \multicolumn{3}{l}{ GLS } \\
\cline { 2 - 7 } FC3 $\leftarrow$ FC1 & No estandarizado & Estandarizado & No estandarizado & Estandarizado & No estandarizado & Estandarizado \\
\hline FC2 $\leftarrow$ FC1 & $-0,006$ & $-0,006$ & $-0,022$ & $-0,023$ & 0,021 & 0,022 \\
\hline FC2 $\leftarrow$ FC3 & 0,077 & 0,051 & 0,089 & 0,062 & 0,134 & 0,088 \\
\hline FC4 $\leftarrow$ FC1 & 0,916 & 0,422 & 0,692 & 0,479 & 0,694 & 0,438 \\
\hline FC4 $\leftarrow$ FC3 & $-0,017$ & 0,760 & 0,992 & 0,780 & 0,925 & 0,812 \\
\hline FC4 $\leftarrow$ FC2 & 0,017 & $-0,014$ & $-0,005$ & $-0,004$ & $-0,012$ & $-0,010$ \\
\hline Modelo de covarianzas & ML & 0,021 & 0,003 & 0,003 & 0,022 & 0,029 \\
\cline { 2 - 7 } & No estandarizado & Estandarizado & No estandarizado & Estandarizado & No estandarizado & Estandarizado \\
\hline FC1 <-> FC2 & 0,036 & 0,049 & 0,035 & 0,052 & 0,067 & 0,099 \\
\hline FC1 <-> FC3 & $-0,003$ & $-0,006$ & $-0,010$ & $-0,023$ & 0,009 & 0,022 \\
\hline FC1 <-> FC4 & 0,455 & 0,761 & 0,467 & 0,781 & 0,412 & 0,816 \\
\hline FC2 <-> FC3 & 0,314 & 0,422 & 0,320 & 0,478 & 0,288 & 0,439 \\
\hline FC2 <-> FC4 & 0,047 & 0,052 & 0,036 & 0,042 & 0,082 & 0,106 \\
\hline FC3 <-> FC4 & $-0,006$ & $-0,009$ & $-0,012$ & $-0,020$ & 0,010 & 0,021 \\
\hline Param & & & ULS &
\end{tabular}

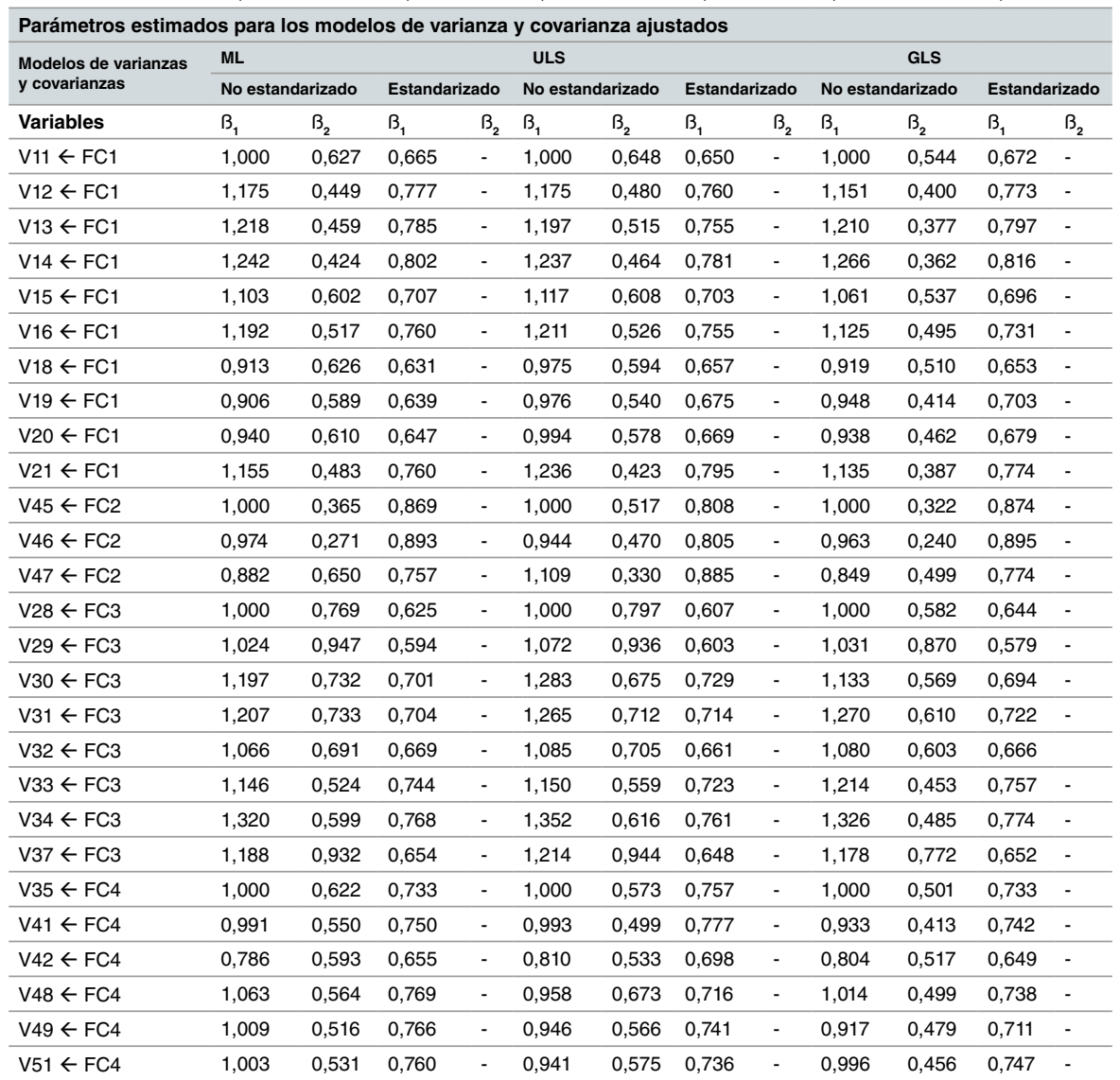

$\boldsymbol{B}_{1}=$ varianza de la variable en relación con su factor para el modelo de medida

$\boldsymbol{B}_{\mathbf{2}}=$ error estimado de la varianza

Nota $=$ el error $B_{2}$ no se estandariza

Fuente: elaboración propia 


\section{Modelo de medida y estructural}

En (1) y (2) se muestra la estructura de las ecuaciones que definen el modelo de medida () para cada una de las variables y modelo estructural () para factores o dimensiones latentes del modelo de clima organizacional.

$$
\begin{aligned}
& \mathrm{Y}_{n}=\beta_{1} X_{n}+\varepsilon_{1} \\
& \mathrm{Y}_{N}=\Upsilon_{1} \xi_{n}+\zeta_{1}
\end{aligned}
$$

Donde:

$\mathrm{n}=$ Variable observada del modelo de medida

$\mathrm{N}=$ Factor dependiente del modelo estructural

$\beta 1$ = Varianza de la variable en relación con su factor para el modelo de medida

$\gamma_{1}=$ Varianza de peso del factor independiente, sobre el factor dependiente del modelo estructural

$\mathrm{X}_{\mathrm{n}}=$ Factor al que pertenece la variable para el modelo de medida

$\xi_{\mathrm{n}}=$ Factor independiente para el modelo estructural

$\varepsilon_{1}=$ Término de error de la variable en el modelo de medida

$\zeta_{1}=$ Error del factor dependiente en el modelo estructural

En la tabla 4 se detalla el modelo estructural que confirma las relaciones entre los factores y que dan forma al cuestionario de medición del clima organizacional en APS. 
Tabla 4. Cálculo de los estimadores del modelo de ecuaciones estructurales

\begin{tabular}{|c|c|c|c|c|c|c|}
\hline \multicolumn{7}{|c|}{ Estimadores de la variable dependiente organización y desempeño FC4 por el método ML } \\
\hline \multirow[t]{2}{*}{ Factor } & \multicolumn{4}{|l|}{ FC4 } & \multirow{2}{*}{ Error FC4 } & \\
\hline & \multicolumn{2}{|l|}{ No estándar } & \multicolumn{2}{|c|}{ Estandarizado } & & \\
\hline FC1 & \multicolumn{2}{|l|}{0,916} & \multicolumn{2}{|l|}{0,760} & \\
\hline FC2 & \multicolumn{2}{|l|}{0,017} & \multicolumn{2}{|l|}{0,021} & \multicolumn{2}{|l|}{0,303} \\
\hline FC3 & \multicolumn{2}{|l|}{$-0,017$} & \multicolumn{2}{|l|}{$-0,014$} & & \\
\hline \multicolumn{7}{|c|}{$\begin{array}{l}\begin{array}{l}\text { Estimadores de estabilidad laboral FC2 y tensión interna y desarrollo profesional FC3 } \\
\text { por el método ML }\end{array} \\
\end{array}$} \\
\hline \multirow[t]{2}{*}{ Factor } & \multicolumn{2}{|l|}{ FC2 } & \multicolumn{2}{|l|}{ FC3 } & \multirow{2}{*}{ Error FC2 } & \multirow{2}{*}{ Error FC3 } \\
\hline & No estándar & Estandarizado & No estándar & Estandarizado & & \\
\hline FC1 & 0,077 & 0,051 & & & 0,918 & 0,494 \\
\hline FC3 & 0,636 & 0,422 & & & & \\
\hline FC1 & & & $-0,006$ & $-0,006$ & & \\
\hline \multicolumn{7}{|c|}{ Bondad de ajuste para los modelos de varianzas y covarianzas } \\
\hline \multirow{2}{*}{$\begin{array}{l}\text { Métodos de } \\
\text { ajuste }\end{array}$} & \multicolumn{2}{|l|}{$M L$} & \multicolumn{2}{|l|}{ ULS } & \multicolumn{2}{|l|}{ GLS } \\
\hline & No ajustado & ajustado & No ajustado & ajustado & No ajustado & ajustado \\
\hline CMIN/DF & 5,816 & 2,371 & 3,708 & 2,714 & 3,170 & 2,027 \\
\hline CMIN & 1849,554 & 699,340 & 1179,007 & 792,389 & 1008,105 & 591,854 \\
\hline DF & 318 & 295 & 318 & 292 & 318 & 292 \\
\hline RMR & 0,076 & 0,065 & 0,073 & 0,060 & 0,136 & 0,108 \\
\hline GFI & 0,784 & 0,920 & 0,976 & 0,984 & 0,872 & 0,925 \\
\hline AGFI & 0,743 & 0,898 & 0,971 & 0,979 & 0,847 & 0,902 \\
\hline NFI & 0,813 & 0,929 & 0,967 & 0,813 & 0,425 & 0,662 \\
\hline TLI & 0,823 & 0,950 & - & - & 0,457 & 0,743 \\
\hline $\mathrm{CFI}$ & 0,840 & 0,958 & - & - & 0,508 & 0,786 \\
\hline \multicolumn{7}{|c|}{ Ajuste de parsimonia } \\
\hline PGFI & 0,737 & 0,781 & 0,876 & 0,813 & 0,385 & 0,551 \\
\hline PCFI & 0,763 & 0,805 & - & - & 0,460 & 0,654 \\
\hline \multicolumn{7}{|c|}{ Ajuste de error RMSEA } \\
\hline RMSEA & 0,091 & 0,049 & - & - & 0,061 & 0,042 \\
\hline LO 90 & 0,087 & 0,044 & - & - & 0,057 & 0,037 \\
\hline HI 90 & 0,095 & 0,053 & - & - & 0,065 & 0,047 \\
\hline
\end{tabular}

Fuente: elaboración propia

La variable latente endógena dependiente FC4 organización y desempeño depende de la variable exógena independiente FC1 gestión interna y de las variables mediadoras FC2 estabilidad laboral y FC3 tensión interna y desarrollo profesional, respectivamente. Cada uno de estos factores presenta parámetros que ratifican las relaciones conceptuales hipotetizadas. 
En el modelo estructural destaca el coeficiente negativo o inverso que relaciona FC3 Tensión interna y desarrollo profesional con FC4 organización y desempeño (FC4 $\leftarrow$ FC3 $=-0,014)$. En (3) se presenta la ecuación estructural resultante del factor independiente FC4.

$$
F C 4=0,760 F C 1+0,021 F C 2-0,014 F C 3+0,303
$$

Se observa así mismo un coeficiente negativo o inverso que relaciona FC1 gestión interna con FC3 tensión interna y desarrollo profesional (FC4 $\leftarrow$ FC3 = -0,006). En (4) y (5) se muestran las ecuaciones estructurales de los factores FC2 estabilidad laboral y FC3 tensión interna y desarrollo profesional, definidos como dimensiones secundarias

$$
\begin{aligned}
& F C 2=0,051 F C 1+0,422 F C 3+0,918 \\
& F C 3=-0,006 F C 1+0,494
\end{aligned}
$$

El factor gestión interna FC1, por el hecho de tener el carácter de exógeno o independiente, no es sujeto del cálculo de su estimador de error y, en consecuencia, no es posible determinar su respectiva ecuación estructural.

\section{Bondad de ajuste del modelo}

Los índices de bondad de ajuste del análisis de ecuaciones estructurales ESEM (tabla 5) son iguales para los modelos de varianzas y covarianzas, con lo que se verifica homocedasticidad en el análisis de los datos.

Los índices de ajuste incremental CFI y TLI alcanzan los valores más altos, ubicándose por encima de 0,95 puntos en el método ML, lo que permite afirmar que el modelo estimado se asemeja adecuadamente a la realidad. Además, los índices de ajuste absoluto GFI y AGFI muestran valores superiores a 0,9 puntos en todos los métodos de ajuste (ML, ULS y GLS), lo que ratifica el ajuste logrado.

Finalmente, el índice RMSA muestra valores inferiores al límite superior del estándar óptimo. Para el ajuste mediante ML se obtiene un índice de 0,049 y, para el método GLS el índice es de 0,042; ambos se ubican en el rango de aceptación $(\leq 0,05)$. De esta forma se confirman los ajustes realizados mediante análisis de ecuaciones estructurales y se ratifica el modelo especificado. 


\section{Discusión}

Las cuatro nuevas dimensiones validan la estructura psicométrica de los reactivos del cuestionario de clima organizacional CCOV propuesto en el modelo original (12), lo que confirma los ítems persistentes que efectivamente superaron los análisis factoriales más estrictos $(39,45)$ y generaron cuatro factores con alta significación estadística $(44,67)$.

El nuevo cuestionario de clima organizacional validado en atención primaria de salud (CCOV-APS), confirmado mediante cargas factoriales y comunalidades altas $(\geq 0,6),(39,45)$, fue modelado estadísticamente (46) y corroborado mediante ecuaciones estructurales $(62,63)$. Las dimensiones confirmadas $(39,45)$ ratifican la capacidad de análisis del cuestionario $(12)$ y validan la redacción y estructura semántica de los reactivos $(1,5,10)$ aplicables en APS $(12,13)$.

Los parámetros que relacionan los factores del clima organizacional (varianzas y covarianzas) $(50,62,63)$ corroboran $(49,65)$ las aportaciones que cada dimensión realiza a un constructo multidimensional complejo $(4,5,7,12)$ del clima organizacional para las entidades de salud.

En general, como se demuestra en las ecuaciones estructurales, se presentan relaciones normales, positivas y directas $(39,41)$ entre los factores estabilidad laboral, gestión interna y organización y desempeño. Se observa, sin embargo, que el factor tensión interna y desarrollo profesional, dada la naturaleza de su comportamiento $(64,65)$, establece relaciones normalmente inversas con los factores gestión interna y organización y desempeño (51).

Por otro lado, el modelamiento confirmado por los respectivos índices de bondad de ajustes $(50,62,63)$ indican que el modelo se acerca lo más posible al modelo real $(44,49,70)$, lo que concuerda con la teoría respecto de las relaciones prestablecidas por el modelo teórico de clima organizacional sometido a evaluación (12).

Por último, el modelamiento realizado confirma relaciones de causalidad entre las dimensiones $(53,54,55)$, ratifica la hipótesis planteada entre los factores (12) y asegura que los tres factores considerados dentro del modelo como dimensiones independientes, tipo espejo o mediadoras $(50,62,63)$, incorporan los planteamientos teóricos que sostienen el modelo $(7,8,12)$. 


\section{Referencias}

1. Cardona DR, Zambrano R. Revisión de instrumentos de evaluación de clima organizacional. Estudios Gerenciales; 2014;30(131):184-9. doi: https://doi.org/10.1016/j.estger.2014.04.007

2. Santa Eulalia J, Sampedro B. Clima organizacional en instituciones de atención primaria de salud. Revista Médica Electrónica. 2012 [citado 2018 ago. 6];34(5):606-19. Disponible en: https://goo.gl/Y7G6d8

3. Silva R, de la Torre J, López A, Bastos S. El clima organizacional en el diseño del Balanced Scorecard: evaluación psicométrica de un instrumento de medida. Revista Contabilidade Vista \& Revista. 2011;22(1):107-41. Disponible en: https:// www.redalyc.org/pdf/1970/197017517005.pdf

4. Carmona V, Jaramillo E. Estudio del clima organizacional en la E.S.E. Hospital San Jorge de Pereira [tesis para obtención de grado]. Pereira, Colombia: Universidad Tecnológica de Pereira; 2010.

5. Pelaes O. Relación entre el clima organizacional y la satisfacción del cliente en una empresa de servicios telefónicos. Universidad Nacional Mayor de San Marcos. Programa Cybertesis PERÚ, [Internet] 2010 [citado 2018 ago. 6]. Disponible en: https://goo.gl/MZ5sxZ

6. Cújar A, Ramos C, Hernández H, López J. Cultura organizacional: evolución en la medición. Estudios Gerenciales. 2014;29(128):350-5. Disponible en: https:// www.redalyc.org/articulo.oa?id=21229786009

7. Likert R. Una técnica para medir actitudes. Nueva York: Trillas; 1976.

8. Halpin A, Crofts D. The organizational climate of schools. International Review of Education. 1963;22(4):441-63. Disponible en: https://ink.springer.com/article/10.1007/BF00598815

9. Litwin G, Stringer R. Motivation and organizational climate. Boston: Harvard University Press; 1968.

10. Pérez de Maldonado I, Maldonado M, Bustamante S. Clima organizacional y gerencia: inductores del cambio organizacional. Investigación y Postgrado [internet]. 2006 [citado 2018 ago. 6];21(2):231-48. Disponible en: https://goo.gl/TdWcV3

11. Segredo Pérez AM. Clima organizacional en la gestión del cambio para el desarrollo de la organización. Rev Cubana Salud Pública [Internet]. 2013 [citado 2018 ago. 6];39(2):385-93. Disponible en: https://goo.gl/onArbA

12. Bustamante MA, Lapo MC, Grandón ML. Creación de un cuestionario de clima organizacional para hospitales de alta complejidad, Chile. Gerencia y Políticas de Salud [Internet]. 2016 [citado 2018 ago. 6];15(30). Disponible en: https://goo. $\mathrm{gl} / \mathrm{yYZDRX}$ 
13. Bustamante MA, Grandón ML, Lapo MC. Caracterización del clima organizacional en hospitales de alta complejidad en Chile. Estudios Gerenciales [Internet] 2015. [citado 2018 ago. 6];31(137):432-40. Disponible en: https://goo.gl/mGZMxw

14. González-Burboa A, Manríquez C, Venegas M. Clima organizacional en una dirección de administración de salud municipal. Ciencia \& Amp Trabajo. 2014;16(51):152-7. Disponible en: https://scielo.conicyt.cl/pdf/cyt/v16n51/art05.pdf

15. Poghosyan L, Nannini A, Clarke S. Organizational climate in primary care settings: implications for nurse practitioner practice. Journal of the American Association of Nurse Practitioners [Internet]. 2013 [citado 2018 ago. 6];25(3):13440. Disponible en: https://goo.gl/nxcjfh

16. Bernal I, Pedraza N, Sánchez M. El clima organizacional y su relación con la calidad de los servicios públicos de salud: diseño de un modelo teórico. Revista Estudios Gerenciales. 2015;31(134):8-19. doi: 10.1016/j.estger.2014.08.003

17. Patarroyo JC. Clima organizacional: elemento clave para el proceso de calidad en las instituciones prestadoras de salud (other). Universidad Nacional de Colombia [Internet]. 2012 [citado 2018 ago. 6]. Disponible en: https://goo.gl/GXEGMC

18. Segredo A, García A, López P, Cabrera P, Perdomo I. Enfoque sistémico del clima organizacional y su aplicación en salud pública. Rev Cub Salud Pública. 2015;41(1):115-29. Disponible en: http://scielo.sld.cu/scielo.php?script=sci_arttext\&pid=S0864-34662015000100010

19. Carlfjord S, Andersson A, Nilsen P, Bendtsen P, Lindberg M. The importance of organizational climate and implementation strategy at the introduction of a new working tool in primary health care. Journal of Evaluation in Clinical Practice [Internet]. 2010 [citado 2018 ago. 6];16(6):1326-32. Disponible en: https://goo.gl/BB6QCN

20. Vignolo J, Vacarezza M, Álvarez C, Sosa A. Niveles de atención, de prevención y atención primaria de la salud. Archivos de Medicina Interna [Internet]. 2011 [citado 2018 ago. 6];33(1):7-11. Disponible en: https://goo.gl/UE8FRj

21. OMS. El informe: Más sano, más justo, más seguro: la travesía de la salud mundial 2007-2017 [Internet]. 2017 [citado 2018 ago. 6]. Disponible en: http:// www.who.int/es/

22. Ministerio de Salud de Chile. Informe sobre brechas de personal de salud por servicio de salud. (n. d.). Santiago, 31 de marzo de 2016 [Internet]. 2016 [citado 2018 ago. 6]. Disponible en: https://goo.gl/eEEwdR

23. Bass del Campo C. Family health model in Chile and greater resoluteness of primary health care: contradictory or complementary? Medwave [Internet]. 2012 [citado 2018 ago. 6];12(11):e5571-71. Disponible en: https://goo.gl/vdhmws

24. Noguera JR, Samudio M. Diagnóstico del clima organizacional del Hospital Central de las Fuerzas Armadas de Paraguay. Mem Inst Investig Cienc Salud. 2014;12(1):1425. Disponible en: http://scielo.iics.una.py/pdf/iics/v12n1/v12n1a04.pdf 
25. Ivancevich JM, Lyon HL. Organizational climate, job satisfaction, role clarity and selected emotional reaction variables in a hospital milieu. Lexington, Kentucky: Office of Development Services, University of Kentucky; 1972.

26. Neal A, Griffin M, Hart P. The impact of organizational climate on safety climate and individual behavior. Safety Science. 2000;34:99-109. Disponible en: http://158.132.155.107/posh97/private/behavioral-safety/organizational-climate-Neal.pdf

27. Vega D, Arévalo A, Sandoval J, Aguilar M, Giraldo J. Panorama sobre los estudios de clima organizacional en Bogotá, Colombia. Revista Diversitas Perspectivas en Psicología. 2006;2(2):329-49. Disponible en: https://www.redalyc.org/ html/679/67920212/

28. Bustamante MA, del Río MC, Lobos GE, Villarreal PI. Percepción de la motivación de directivos intermedios en tres hospitales complejos de la Región del Maule, Chile. Salud Pública Mex. 2009;51(5):417-26. Disponible en: https://www. redalyc.org/pdf/106/10612565008.pdf

29. Salinas C, Laguna J, Mendoza M. La satisfacción laboral y su papel en la evaluación de la calidad de la atención médica. Salud Pública Mex. 1994;36(1):22-9. Disponible en: https://www.redalyc.org/pdf/106/10636105.pdf

30. Hernández V, Quintana L, Guedes R, Mederos R, Sablón N. Estudio de la motivación, la satisfacción, la cultura organizacional y la calidad del servicio existente en el Hospital Mario Muñoz Monroy. Folletos Gerenciales. 2006;1(10):37-57. Disponible en: https://www.icesi.edu.co/revistas/index.php/estudios_gerenciales/ article/view/1920/2482

31. Delgado A, Bellón J, Martínez M, Luna J, López L, Lardelli P. Las dimensiones del clima organizacional percibidas por los médicos de familia. Aten Primaria. 2006;37(9):489-97. Disponible en: https://core.ac.uk/download/pdf/82156052.pdf

32. Bermejo M, Bermejo G, de la Rosa G, Montaudon BG. Evaluación del clima organizacional en una unidad hospitalaria de tercer nivel. Rev Cir (Mex). 2008;75(1):50-7. Disponible en: http://www.medigraphic.com/cgi-bin/new/resumenI.cgi? IDARTICULO $=42160$

33. Aldana O, Hernández M, Aguirre D, Hernández S. Clima organizacional en una unidad de segundo nivel de atención. Revista de Enfermería del Instituto Mexicano del Seguro Social [Internet]. 2009 [citado 2018 ago. 6];(17):91-6. Disponible en: https://goo.gl/6p2TUk

34. Greenslade J, Jimmieson N. Organizational factors impacting on patient satisfaction: A cross sectional examination of service climate and linkages to nurses' effort and performance. Int J NursStud. 2011;48(10):1188-98. doi: 10.1016/j. ijnurstu.2011.04.004. 
35. Segura A. Clima organizacional: un modo eficaz para dirigir los servicios de salud. Avances en Enfermería. 2012;30(1):107-13. Disponible en: https://revistas. unal.edu.co/index.php/avenferm/article/view/35442/36514

36. De Araujo SR, de la Torre J, López A, Bastos S. El clima organizacional en el diseño del Balanced Scorecard: Evaluación psicométrica de un instrumento de medida. Revista Contabilidade Vista \& Revista [Internet]. 2011 [citado 2018 ago. 6]; 22(1):107-41. Disponible en: https://goo.gl/d77YvD

37. Bernal, I, Pedraza NA, Sánchez I. El clima organizacional y su relación con la calidad de los servicios públicos de salud: diseño de un modelo teórico. Estudios Gerenciales. 2015 [citado 2018 ago. 6];31:8-19. Disponible en: https://goo.gl/ XDggRo

38. García A, Moro M, Medina M. Evaluación y dimensiones que definen el clima y la satisfacción laboral en el personal de enfermería. Revista de Calidad Asistencial. 2010;25(4):207-14. Disponible en: https://dialnet.unirioja.es/servlet/ articulo? codigo $=3321517$

39. Rodríguez A, Álvarez A, Sosa I, De Vos P, Bonet MH, Van der Stuyft P. Inventario del clima organizacional como una herramienta necesaria para evaluar la calidad del trabajo. Revista Cubana de Higiene y Epidemiología [Internet]. 2010 [citado 2018 ago. 6];48(2):177-96. Disponible en: https://goo.gl/tL4awQ

40. Izquierdo I, Olea J, Abad FJ. Exploratory factor analysis in validation studies: Uses and recommendations. Psicothema [Internet]. 2014 [citado 2018 ago. 6];26(3):395-400. Disponible en: https://goo.gl/Kemkqr

41. Ferrando P, Anguiano-Carrasco C. El análisis factorial como técnica de investigación en psicología. Papeles del Psicólogo [Internet]. 2010 [citado 2018 ago. 6];31(1). Disponible en: https://goo.gl/nAKzHr

42. Beavers AS, Lounsbury JW, Richards JK, Huck SW, Skolits GJ Esquivel SL. Practical considerations for using exploratory factor analysis in educational research. Pract Assess Res Eval. 2013;18(6). Disponible en: http://pareonline. net/pdf/v18n6.pdf

43. Hernández R, Fernández C, Baptista P. Metodología de la investigación, 5. ${ }^{a}$ ed. México D. F.: McGraw-Hill; 2010 [citado 2018 ago. 6]. Disponible en: https:// goo.gl/q1qEu8

44. Eyssautier M. Metodología de la investigación. Desarrollo de la inteligencia, 5. a ed. México: Thomson; 2006 [citado 2018 ago. 6]. Disponible en: https://goo.gl/nuvh85

45. Guarín A, Ramírez A, Torres F. Modelos multinomiales: un análisis de sus propiedades. Revista Ingenierías Universidad de Medellín [Internet]. 2012 [citado 2018 ago. 6];11(20):87-104. Disponible en: https://goo.gl/k3bzss

46. Abad FJ, Olea J, Ponsoda J, García C. Medición en ciencias sociales y de la salud. Madrid: Síntesis; 2011 [citado 2018 ago. 6]. Disponible en: https://goo.gl/H2LeAb 
47. Matsunaga M. How to factor-analyze your data right: Do's, don'ts, and how-to's. International Journal of Psychological Research [Internet]. 2010 [citado 2018 ago. 6];3(1):97-110. Disponible en: https://goo.gl/CjnpUm

48. Edwards JR. The fallacy of formative measurement. Organizational Research Methods; 2011;14(2):370-88. Disponible en: https://journals.sagepub.com/ doi/10.1177/1094428110378369

49. Baño Ayala DJ, Villacrés C. EP, Arboleda Á. LF, García Zapata T. Evaluación del clima organizacional del personal docente de las Instituciones de Educación Superior (IES) de la provincia de Chimborazo - Ecuador. Revista Industrial Data. 2016;19(2):59-68. Disponible en: http://revistasinvestigacion.unmsm.edu. pe/index.php/idata/article/view/12816

50. Lloret-Segura S, Ferreres-Traver A, Hernández-Baeza A, Tomás-Marco I. El análisis factorial exploratorio de los ítems: una guía práctica, revisada y actualizada. Anales de Psicología [Internet]. 2014 [citado 2018 ago. 6];30(3):1151-69. Disponible en: https://goo.gl/HCXJyc

51. Pérez E, Medrano L. Análisis factorial exploratorio: bases conceptuales y metodológicas. Revista Argentina de Ciencias del Comportamiento [Internet]. 2010 [citado 2018 ago. 6];2(1):58-66. Disponible en: https://goo.gl/Kcdqh8

52. Kaplan D. Structural equation modeling: foundations and extensions, $2 .^{\mathrm{a}}$ ed. Thousand Oaks, CA: Sage Publications; 2009.

53. George D, Mallery P. SPSS for Windows step by step: A simple guide and reference. 11.0 update, $4 .^{\mathrm{a}}$ ed. Boston: Allyn \& Bacon; 2003.

54. Kaiser HF. An index of factorial simplicity. Psychometrika. 1974;39:31-6. Disponible en: https://link.springer.com/article/10.1007/BF02291575

55. Borsboom D, Mellenbergh GJ, Van Heerden J. The theoretical status of latent variables. Psychological Review. 2003;110(2):203-19. doi: 10.1037/0033295X.110.2.203.

56. Chión S, Charles V. Analítica para la modelación estructural. Lima: Pearson Perú; 2016.

57. Hair J, Anderson R, Tatham R, Black W. Análisis multivariante, 5. ${ }^{\text {a }}$ ed. Madrid: Prentice Hall; 1999.

58. Escobedo M, Hernández J, Estebané V, Martínez G. Modelos de ecuaciones estructurales: características, fases, construcción, aplicación y resultados. Cienc Trab. 2016; 18(55):16-22. Disponible en: https://scielo.conicyt.cl/pdf/cyt/v18n55/ art04.pdf

59. Kline R. Principles and practice of structural equation modeling. Nueva York: Gilford Press; 2005.

60. Byrme BM. Structural equation modeling whit AMOS. Asic concepts, applications and programming. Mahwah, NJ: Lawrence Eribaum Associates; 2001. 
61. Lévy J, Varela J. Modelación con estructuras de covarianzas en ciencias sociales. Madrid: Netbiblio; 2006.

62. Lévy JP. Modelización y análisis con ecuaciones estructurales. En: Lévy JP, Varela J, editores. Análisis multivariante para las ciencias sociales. Madrid: Prentice Hall; 2003.

63. Morin Alexandre JS, Parker PD. Gurvinder Kaur. Exploratory structural eqation modeling: an integration of the best features of exploratory and confirmatory factor analysis. Supplemental material. Annu Rev Clin Psychol [Internet]. 2014 [citado 2018 ago. 6];10:85-110. Disponible en: https://goo.gl/49gGk5

64. Forero CG, Maydeu-Olivares A, Gallardo-Pujol D. Factor analysis with ordinal indicators: A monte Carlo study comparing DWLS and ULS estimation. Structural Equation Modeling [Internet]. 2009 [citado 2018 ago. 6];16:625-41. Disponible en: https://goo.gl/FTrFBS

65. Flora DB, Labrish C, Chalmers RP. Old and new ideas for da-ta screening and assumption testing for exploratory and confirmatory factor analysis. Frontiers in Quantitative Psychology and Measurement [Internet]. 2012 [citado 2018 ago. 6];3(55):1-21. Disponible en: https://goo.gl/FgiDZV

66. Lee CT, Zhang G, Edwards MC. Ordinary least squares estimation of parameters in exploratory factor analysis with ordinal data. Multivariate Behavioral Research [Internet]. 2012 [citado 2018 ago. 6];47:314-39. Disponible en: https:// goo.gl/hy9hqt

67. Jung S. Exploratory factor analysis with small sample sizes: A com-parison of three approaches. Behavioural Processes [Internet]. 2013 [citado 2018 ago. 6]; 97:90-5. Disponible en: https://goo.gl/wM2Ub5

68. Lorenzo-Seva U, Ferrando PJ. FACTOR 9.2: A comprehensive program for fitting exploratory and semiconfirmatory factor analysis and IRT models. Applied Psychological Measurement [Internet]. 2013 [citado 2018 ago. 6];37(6):497-8. Disponible en: https://goo.gl/RysJ8a

69. Muthen B, Asparouhov T. Bayesian SEM: A more flexible representation of substantive theory. Psychological Methods [Internet]. 2013 [citado 2018 ago. 6];17(3):313-35. Disponible en: https://goo.gl/LwDBBr

70. Muthén B, Kaplan D. A comparison of some methodologies for the factor analysis of non-normal Likert variables. British Journal of Mathematical and Statistical Psychology [Internet]. 1985 [citado 2018 ago. 6];38:171-89. Disponible en: https:// goo.gl/DfmNje 